\title{
Experimental studies and mathematical modeling on the effects of rapid airflow and baking temperature during baking
}

\begin{abstract}
Rapid airflow in oven will influence the heat transfer in baking process therefore the purpose of this study is to experimentally and numerically investigate the effects of operating conditions on the heat transfer mechanism and volume expansion during baking. Cakes are baked in an air fryer and convection oven with constant speed $5.11 \mathrm{~m} / \mathrm{s}$ and $0.88 \mathrm{~m} / \mathrm{s}$ respectively at $150,160,170{ }^{\circ} \mathrm{C}$ in different baking times. A heat transfer model was defined to describe the influence of baking temperature on internal cake temperature by Fourier's law. It was observed that the presence of rapid airflow (air fryer) and increment in oven temperature yielded an increase in volume expansion but produced a less moist product. Cakes baked in the presence of rapid airflow at 150 ${ }^{\circ} \mathrm{C}$ were moister but with little volume expansion in the cakes compared to convection oven-baked cakes. Significant correlation between the numerical models with experimental temperature profiles were recorded during complete cake baking process.
\end{abstract}

Keyword: Air fryer; Convection oven; Heat transfer; Oven temperature; Rapid air 